\title{
Selected Abstracts from the I 4th Annual HMO Research Network Conference, April 13-16, 2008, Minneapolis, Minnesota
}

\section{Oral Presentations}

\author{
Research Administration \\ Abstract C-A1-01 \\ Administrative Support for Advancing Collaborative HMORN \\ Research
}

Barry Miller, $\mathrm{PhD}^{1}$, Division of Research, Kaiser Permanente; Ellen Deitch, Center for Health Research, Kaiser Permanente; Robin Hudson, Center for Health Research, Southeast, Kaiser Permanente; Tim O'Bar, Center for Health Studies, Group Health; Ben Prince, Fallon Clinic; Kate Rardin, HealthPartners Research Foundation; Barbara Richard, Harvard Pilgrim; Paul Steele, Center for Health Research, Kaiser Permanente

${ }^{1}$ Deceased October 17, 2008

Background: Frequently, only a short time exists between preparation of a multi-site HMORN proposal and its submission. When a collaborative project is under development, the prime institution needs to work individually with other institutions to gather information for submission. This approach is significantly time consuming. Methods: Over the past year and a half, the above presenters have been addressing ways in which collaborative HMORN grant submissions and awards could be streamlined and facilitated. This working group has begun to address such issues as: developing a web site in order to house HMORN members' resources, boilerplate documents, guidelines, etc.; effort reporting practices; standardizing checklists and statement of intent forms; compiled human subjects training requirements; facilitating the implementation of multi-site clinical trials; and developing post award subcontract boilerplates. Several working committees were established and convened on a monthly basis to address each of these issues. Each committee was charged with the responsibility of creating guidelines and/or documents that would successfully address these issues. Results: The results of this year's efforts will be presented at this workshop. Materials that have been created will be distributed to all audience participants and subsequently posted on the HMORN website. In addition, all attendees will be asked for additional input to the final recommendations of the committees. Lastly, participants at this workshop will be asked to indicate those issues that, as administrators, they believe will continue to need to be addressed in order to further facilitate collaborative grant applications among the HMORN members. Conclusions: Multiple multi-site grants have been submitted and awarded to one of the HMORN members on behalf of the HMORN. The CRN, CERT, CCSN, IDSRN, as well as the Vaccine Data Safety Link, the Cancer Care and Outcomes Research Surveillance Consortium and, most recently, the Cardiovascular Research Network are examples. In a tight funding environment, additional collaborative grant applications will become increasingly necessary. Success can be further enhanced with streamlined research administration practices.

\section{Pharmacoepidemiology}

Abstract C-A2-02

Development, Implementation and Evaluation of an Educational Curriculum on Pharmaceutical Marketing and Prescribing

David W. Price, MD, CPMG Dept of Education/Kaiser Permanente Colorado Institute of Health Research; Marsha A. Raebel, PharmD, Kaiser Permanente
Colorado Institute of Health Research; Douglas A. Conner, PhD, Kaiser Permanente Colorado Institute of Health Research; Leslie A. Wright, MA, Kaiser Permanente Colorado Institute of Health Research

Background: Lawsuit settlement funds from off-label marketing of Neurontin were earmarked for clinician education on drug development, approval and marketing. Four HMORN CERTs sites, including Kaiser Permanente Colorado (KPCO), received grants. Aims: To develop, implement, and evaluate curricula on drug marketing and prescribing based on needs assessments of prescribing clinicians (PCs) and KPCO organizational leaders. Methods: Online interactive curricula were developed after surveys of physicians, nurse practitioners, physician assistants and KPCO organizational leaders. 'Pharmaceuticals from Development to Practice,' included 3 modules (4 CME credits): Off-Label Use of Pharmaceuticals, Accessing \& Appraising Unbiased Drug Information, and Addressing Patient Inquiries about Specific Medications Advertised to Consumers. Modules contained didactic material, case studies incorporating heavily marketed medications, and self-reflection questions. Participants could pose questions to faculty, who responded by email. Participants were invited to a follow-up session to share learnings and interact with faculty. PCs were randomized to participate in 2 phases, the latter cohort a delayed comparison group for prepost time-series prescribing analysis of heavily marketed medications. Other outcomes included self-reported intent to change, knowledge changes, number and descriptions of questions posed to faculty, qualitative summaries of reflection questions, and participant ratings of each module. Results: Final sample sizes were 43 for prescribing and 47 for other outcomes. Each module had 33 to 44 participants. 14 questions were posed to faculty; 131 intent-tochange statements were generated. Nearly all participants agreed that the modules met their objectives. Over $90 \%$ of participants indicated increased knowledge on accessing/appraising drug information and off-label prescribing/promotion; $70 \%$ indicated increased comfort in addressing patient inquiries. Due to small sample size, cohorts will be combined for prescribing analysis, with participants compared to non-participants. Conclusions: Participants valued the curricula on the impacts of pharmaceutical marketing and prescribing. Subsequent evaluation will include effect on prescribing and follow-up on intended practice changes. Additional strategies are needed to increase prescriber interest and participation in curricula on this topic.

Abstract C-A2-04

Adherence to Urate Lowering Medications for the Treatment of Chronic Gout

Leslie R. Harrold, MD, MPH, Meyers Primary Care Institute/Fallon Clinic; Becky Briesacher, PhD, Meyers Primary Care Institute/Fallon; Marsha Raebel, Pharm D, Kaiser Permanente Colorado; Hassan Fouayzi, MS, Meyers Primary Care Institute/Fallon; Robert Yood, MD, Meyers Primary Care Institute/Fallon; Susan E. Andrade, ScD, Meyers Primary Care Institute/Fallon

Background: Patients with chronic gout require sustained treatment with urate-lowering drugs (ULDs). Little is known regarding adherence to medication treatment for gout. Our aim was to assess the level and determinants of adherence with ULDs prescribed for chronic gout. Methods: A retrospective cohort study was conducted using administrative data from two health plans participating in the HMO Research Network Center for Education and Research on Therapeutics. We identified all persons 18 years 
or older with a diagnosis of gout who initiated use of a ULD (allopurinol or probenecid) from January 1, 2000 through June 2006 who were enrolled for at least 12 months after the first ULD dispensing. Adherence was measured based on the medication possession ratio (MPR) which was determined by the sum of the day's supply of drug therapy from the first dispensing to the end of the 12-month period. Covariates included age, sex, comorbid illnesses and medications associated with gout, concurrent treatments for gout and the Charlson comorbidity index. Results: Preliminary data for 627 gout patients at one health plan indicated the median MPR was $0.49( \pm 0.32)$ for allopurinol and $0.48( \pm 0.37)$ for probenecid. Factors associated with poor adherence (defined as an MPR $<0.80$ ) included younger age $(<45 \mathrm{yrs})$, fewer comorbid conditions, use of nonsteroidal anti-inflammatory drugs (NSAIDs) prior to initiation of a ULD, and use of a glucocorticoid or NSAID after initiating a ULD. Conclusions: Nonadherence is common in gout, particularly in young men without other comorbidities. In addition, it appears those with more active gout (based on dispensings of NSAIDs and glucocorticoids) are less adherent.

\section{Abstract C-A2-05 \\ Pneumonia Risk Among COPD Patients Using Fluticasone/Salmeterol Versus Other Inhaled Steroids and Bronchodilators Alone}

Michael Schum, PhD, Lovelace Clinic Foundation; Douglas W. Mapel, MD, MPH, Lovelace Clinic Foundation; Jeff Brown, PhD, Department of Ambulatory Care and Prevention, Harvard Pilgrim Health Care/Harvard Medical School; Marianne U. Yood, DSc, Yale University School of Medicine, Henry Ford Health System; David P. Miller, ScD, GlaxoSmithKline Research and Development; Kourtney J. Davis, PhD, GlaxoSmithKline Research and Development

Background/Aims: In recently published randomized clinical trials, COPD patients using inhaled corticosteroids (ICS) alone or in combination with long-acting beta-agonists (LABA) were found to have a small but significantly increased risk of pneumonia. The purpose of this project was to determine whether general population COPD patients using a combined fluticasone and salmeterol inhaler had a higher risk for pneumonia compared to those using other inhaled ICS or bronchodilators, either alone or in combination. Methods: COPD patients were identified from three health systems in different regions in the United States. The study population was comprised of 5245 individuals who used some form of inhaled treatment during the study period (September 1, 2000 through August 31, 2003), 2154 of whom had at least one pneumonia episode confirmed by chest X-ray or hospitalization. Nested case-control methods were used to calculate odds ratios (OR) for the risk of pneumonia while on therapy. Results: With patients using short-acting bronchodilators as the reference group, the only treatment associated with a possibly increased risk of pneumonia was ICS used alone (OR, 1.29; 95\% CI, $0.96-1.73 ; P=0.09)$. Users of LABA alone (OR, $0.92 ; 95 \% \mathrm{CI}, 0.69-1.22)$ and fluticasone and salmeterol in combination (OR, 1.03; 95\% CI, 0.74-1.42) had no increased risk for pneumonia. Conclusions: In this retrospective analysis of a large COPD cohort, treatment with any ICS, LABD, or ICS and LABD in combination was not associated with a substantially increased risk for developing pneumonia. Funded By: GlaxoSmithKline Research \& Development.

\section{Abstract C-A2-08 \\ Prescribing to Older Individuals in Conjunction With Emergency Department Visits}

Marsha A. Raebel, PharmD, Kaiser Permanente Colorado Institute for Health Research; Laurie Crounse, MPH, Kaiser Permanente Colorado Institute for Health Research; Gwyn Saylor, BA, Kaiser Permanente Colorado Institute for Health Research

Background/Aims: The emergency department (ED) is a high-risk care setting. Risk of adverse drug events (ADE) associated with ED care is insufficiently explored, particularly among older patients. We examined prescribing to individuals aged 60 years or older during an ED visit. The aims were to (1) ascertain drugs newly initiated in conjunction with an ED visit, (2) determine the proportion of newly initiated drugs that are considered high-risk, (3) determine the proportion of patients with discharge diagnoses associated with increased ADE risk, and (4) describe repeat ED visits among these patients. Methods: Patients must have had health plan membership for
6 months prior to the ED visit and remained members (or die) within 6 months after the visit. Data to identify the cohort, determine prescribing, and establish ED visits were extracted from electronic medical records and administrative claims. Drugs were defined as newly initiated if no drug within that therapeutic class was dispensed to that patient within the previous 180 days and if the drug was dispensed within 72 hours of ED discharge or before the next outpatient visit (if sooner). Drugs were classified as high-risk based on literature associating the drugs with ADE (i.e., drug-drug or drug-disease interactions, drug-laboratory monitoring recommendations, drugs to avoid in the elderly). Discharge diagnoses were classified as increased risk of ADE based on published evidence. A repeat ED visit was defined as an ED visit within 180 days of the first ED visit. Results: At Kaiser Permanente Colorado in 2006, 6868 older patients were discharged to home after an ED visit, with 1338 patients newly initiated on 1883 drugs. Ten classes accounted for $80 \%$ of newly initiated drugs; all are high-risk for ADE among older patients: narcotics $(27 \%)$, antibiotics $(25 \%)$, corticosteroids $(6 \%)$, antihistamine/cold remedies (5\%), antispasmodics (4\%), anti-anxiety agents $(4 \%)$, anti-asthmatics $(3 \%)$, anticoagulants $(2 \%)$, gastrointestinal drugs $(2 \%)$, and muscle relaxants $(2 \%)$. A primary discharge diagnoses was listed in $25 \%$ of visits; diagnoses included cardiovascular, respiratory, gastrointestinal, neurologic, metabolic, end-stage renal disease, hypoglycemia, hemorrhage, rash, and syncope. $48 \%$ of patients had a repeat ED visit. Conclusions: Among older patients, high-risk drugs are often initiated in conjunction with an ED visit. Work is needed to determine the extent to which drugs initiated during $\mathrm{ED}$ visits result in $\mathrm{ADE}$.

\section{Abstract C-A2-09 \\ Older Age and Less Aggressive Hypoglycemic Therapy for Diabetes Mellitus}

Jennifer Tjia, MD, MSCE, University of Massachusetts Medical School; Susan Andrade, PhD, University of Massachusetts Medical School

Background: Pharmacoepidemiologic studies using claims databases show lower intensity of drug treatment for diabetes mellitus (DM) with older age and greater comorbidity, but do not account for level of glycemic control. The aim of this study is to describe patterns of hypoglycemic use among older adults with DM while controlling for patient-level factors, including glycemic control. Methods: We conducted a cross-sectional study among enrollees of a mixed-model, not-for-profit HMO operating in New England. We identified 772 patients who were aged 65 years and older, had a diagnosis of type 2 diabetes mellitus from January 1 to December 31, 2002, and used at least one hypoglycemic medication. Information on age, sex, prescription drug dispensings, inpatient and outpatient diagnoses and procedures, and laboratory test results were obtained from HMO automated databases. Comorbidity burden was assessed by calculating the Chronic Disease Score based on prescription drug dispensings. We used multivariable logistic regression to identify the independent effect of age on intensity of hypoglycemic medication use (monotherapy vs multidrug or insulin therapy) while controlling for patient-level factors including glycemic control, Chronic Disease Score, and presence of coronary heart disease (CHD), hypertension and dyslipidemia. Results: Overall, $541(70.1 \%)$ used one oral hypoglycemic agent, $181(23.5 \%)$ used two oral agents, $10(1.3 \%)$ used three oral agents, and $26(3.4 \%)$ used insulin therapy. In adjusted analysis, predictors of less aggressive (monotherapy) versus more aggressive (multidrug or insulin) therapy were older age ( $=85$ yrs vs $65-74$ yrs; odds ratio, 0.32 ; 95\% CI, 0.15-0.67) and prior hospitalization (OR, 0.45; 95\% CI, $0.25-0.80)$. Poorer glycemic control was associated with more aggressive hypoglycemic therapy ( $\mathrm{HgAl} 1 \mathrm{c}>9$ vs $\mathrm{HgAl} \mathrm{c}<7$; OR, 2.9; 95\% CI, 1.3-6.5). History of CHD increased the likelihood of using more aggressive hypoglycemic therapy (OR, 1.6; 95\% CI, 1.06-2.45). Conclusions: Although prevalence and risk of complications from diabetes increases with age, intensity of drug treatment for diabetes declines with older age even after adjustment for glycemic control and comorbidity. 